\title{
A universal strategy for the synthesis of porous two- dimensional transition metal oxide nanosheets based on chemical topology transformation
}

\author{
Xiang $\mathrm{Li}^{1}$, Ziyu Qin ${ }^{2}$, Xiafen $\mathrm{Hu}^{1}$, Jian Zhang ${ }^{3}$, Jinjin $\mathrm{Wu}^{4}$, Huimin Yang ${ }^{1}$, Changsheng Xie ${ }^{1}$ and \\ Dawen Zeng ${ }^{*}$
}

\begin{abstract}
Two-dimensional (2D) transition metal oxides (TMOs) have attracted much attention for various applications, owing to the abundance of active sites, rapid ion transmission speed, and short carrier migration distance. However, the current preparation strategies are usually limited to producing intrinsically layered compounds or sacrificing template. Here, we report a universal strategy for preparing ultrathin porous 2D TMO nanosheets by chemical topological transformation of the corresponding transition metal selenides. We observed that the as-prepared 2D TMO nanosheets not only perfectly inherit the transition metal selenides' 2D topological structure, but also possess numerous pore structures formed as a way to release the structural stress associated with lattice growth. As a proof of concept, ultrathin porous $\mathrm{WO}_{3}, \mathrm{MoO}_{3}$, and $\mathrm{Co}_{3} \mathrm{O}_{4}$ nanosheets were successfully prepared based on the in-situ oxidation of the corresponding ultrathin $\mathrm{WSe}_{2}, \mathrm{MoSe}_{2}$, and $\mathrm{Co}_{0.85} \mathrm{Se}$, respectively. The outstanding sensing properties and photodetector performance displayed by the as-prepared porous $2 \mathrm{D} \mathrm{WO}_{3}$ nanosheets further indicate the promising prospects of topology transformation for the preparation of porous 2D TMO nanosheets.
\end{abstract}

Keywords: topology transformation, two-dimensional nanosheets, porous, transition metal oxide

\section{INTRODUCTION}

Graphene discovered in 2004 [1] has sparked a worldwide boom in research on two-dimensional (2D) materials as a result of graphene's unique physical, chemical, and electrical properties. Many ultrathin materials with graphenelike structures, such as transition metal oxides (TMOs) [2], transition metal chalcogenides [3], graphitic carbon nitride [4], and layered double hydroxides [5], have attracted widespread attention and spurred a range of research efforts. Ultrathin 2D nanosheets usually have a lateral dimension of $100 \mathrm{~nm}$ or larger and a thickness of only a few $\mathrm{nm}[6]$; in addition to having a large specific surface area, ultra-high carrier mobility, good electrical conductivity, and excellent mechanical properties, research results also indicate that $2 \mathrm{D}$ materials can confine electrons in a $2 \mathrm{D}$ space, which affords surprising performance in many applications [7]. In recent years, the continuous development of the semiconductor industry has meant that higher performance and economic benefit requirements now exist for functional materials; in this context, TMOs have attracted increasing attention as a class of compounds comprising elements that are extremely abundant on earth. Besides the economic advantages derived from the abundance of transition metals on earth, 2D TMOs are also considered functional materials with very high application potential due to their unique mixed valence, rich redox combinations, rapid ion transmission speed, short carrier migration distance, and high-temperature stability. Results from published studies indicate that 2D TMOs display ideal performances in the

\footnotetext{
${ }^{1}$ The State Key Laboratory of Materials and Processing Die \& Mould Technology, Department of Materials Science and Engineering, Huazhong University of Science and Technology, Wuhan 430074, China

${ }^{2}$ Hainan Provincial Fine Chemical Engineering Research Center, Hainan University, Haikou 570228, China

${ }^{3}$ Key Laboratory of Optoelectronic Devices and Systems of Ministry of Education and Guangdong Province, College of Optoelectronic Engineering, Shenzhen University, Shenzhen 518060, China

${ }^{4}$ Analysis and Testing Center, Huazhong University of Science and Technology, Wuhan 430074, China

* Corresponding author (email: dwzeng@mail.hust.edu.cn)
} 
fields of sensors [8-9], catalysis [10], and electrochemical energy storage [11]. These excellent performance and broad application prospects have thus encouraged researchers to develop new strategies for the preparation of 2D TMO nanosheets [12]. However, developing efficient methods to prepare $2 \mathrm{D}$ TMOs remains a challenging research goal.

Generally, the preparation strategies of 2D TMO nanosheets can be divided into two categories: "top-down" and "bottom-up." The peeling method is a typical topdown preparation strategy. The principle on which it is based is to overcome the weak van der Waals forces between the layers of the multilayer material to achieve nanosheet separation. For instance, Ji et al. [13] prepared ultrathin $\mathrm{MoO}_{3}$ nanosheets with a thickness of $10 \mathrm{~nm}$ by affecting the intercalation of dimethyl sulfoxide and the ultrasonic grinding of $\mathrm{MoO}_{3}$ samples of higher thickness. Zhao et al. [14] also prepared several ultrathin $\mathrm{CrO}$ and $\mathrm{ZrO}$ nanosheets by heating and stripping the corresponding metal chloride precursor. However, the accurate control of the thickness and number of TMO layers is very difficult to achieve by implementing the heating and stripping approach, and this method is only suitable for peeling intrinsically layered materials. As for the bottomup synthesis methods, the template-based method has been proven to be a promising strategy for the preparation of ultrathin 2D TMOs. Peng et al. [15] reported a universal strategy for the preparation of 2D TMOs that relies on graphene oxide (GO) as a template by which a variety of porous $2 \mathrm{D}$ TMO nanosheets were successfully prepared by removing the GO template. Similarly, Zhao et al. [16] and Sun et al. [17] prepared 2D TMOs using $\mathrm{GO}$ and a polymer as templates, respectively. Although the template-based method can be utilized to efficiently prepare intrinsically non-layered 2D TMOs, the implementation of the template preparation procedure and the post-treatment to remove the template not only make the synthesis process complex, but also may cause the nanostructures to be destroyed. Notably, chemical vapor deposition and physical vapor deposition have also been proven to afford the controllable synthesis of high-quality 2D TMO nanosheets. However, the low productivity and the high energy consumption associated with the implementation of these strategies have drawn criticism [18]. Therefore, considering the disadvantages and inherent limitations of existing 2D TMO preparation methods, the development of new and more widely applicable preparation strategies of $2 \mathrm{D}$ TMO remains a research goal of high significance.

In this work, considering that, by implementing the chemical topological transformation approach, the maintenance and "inheritance" of the precursor's topological structure during the preparation of the target product can be achieved in the absence of an auxiliary template; while the topology transformation of transition metal selenide (TMSe) nanosheets to TMO nanosheets at the $2 \mathrm{D}$ scale has been rarely mentioned, we demonstrated for the first time a universal strategy for the preparation of porous 2D TMO nanosheets based on the chemical topological transformation approach. By oxidizing the corresponding ultrathin TMSe nanosheets at a specific temperature determined by thermogravimetric analysis (TGA) and differential scanning calorimetry (DSC) measurements (Fig. S1), we were able to successfully prepare several porous 2D TMO nanosheets, including $\mathrm{WO}_{3}, \mathrm{MoO}_{3}$, and $\mathrm{Co}_{3} \mathrm{O}_{4}$, by implementing the chemical topological transformation approach. Transmission electron microscopy (TEM) evidence indicated the synthesized TMO nanosheets display a rich and evenly distributed porosity. In addition, taking $\mathrm{WO}_{3}$ nanosheets as an example, atomic force microscopy (AFM) data indicated that the thickness of the nanosheets of this TMO is only $4-5 \mathrm{~nm}$, a value that is highly consistent with the thickness of the nanosheets of the $\mathrm{WSe}_{2}$ precursor [19]. The explanation of the observation that the prepared TMO nanosheets can perfectly maintain the topological morphology of the utilized precursor may be that the precursor prepared by the hydrothermal method has a relatively loose structure, which provides a suitable $2 \mathrm{D}$ space for the breakage of $\mathrm{W}-\mathrm{Se}$ bonds and the formation of $\mathrm{W}-\mathrm{O}$ bonds, and the formation of a uniform pore structure benefits from the need for stress release during lattice growth. On the one hand, the generation of these pores offsets the tendency of the $2 \mathrm{D}$ TMO nanosheets to crack and undergo granulation, thus ensuring the integrity of the $2 \mathrm{D}$ structure of the TMO nanosheets. On the other hand, studies have shown that a porous structure often implies the presence of highly reactive exposed edges $[10,20-21]$; thus, the abundance of pores in the prepared TMO nanosheets will lead to a large specific surface area and a significantly increased number of active sites; moreover, compared with non-porous $\mathrm{WO}_{3}$ nanosheets, density functional theory (DFT) calculations confirmed that more charge is transferred in $\mathrm{W}$ atoms in porous $\mathrm{WO}_{3}$ nanosheets, so that the nanosheets could have superior performance in many electronic applications. In fact, we found that porous $2 \mathrm{D} \mathrm{WO}_{3}$ exhibited excellent performance in both gas sensing and light excitation, which makes us confident about the application prospects of such porous $2 \mathrm{D}$ TMO nanosheets. 


\section{EXPERIMENTATION}

\section{Material synthesis}

All chemicals were used as purchased without further processing. The preparation of ultrathin porous TMO nanosheets can be divided into two steps: the synthesis of ultrathin selenide nanosheets and the in-situ oxidation of the said nanosheets in an oxygen atmosphere. We synthesized ultrathin and dense selenide nanosheets implementing the hydrothermal method according to literature directions [22-24]; we then completed the inheritance of the $2 \mathrm{D}$ morphology from TMSe to TMO, performing an in-situ oxidation.

\section{Preparation of ultrathin porous $\mathrm{WO}_{3}$ nanosheets}

Dimethylformamide $(50 \mathrm{~mL}$, analytical reagent (AR)) were poured into a three-necked flask along with $8 \mathrm{mmol}$ of Se (99\%) powder and $0.2 \mathrm{~g}$ of $\mathrm{NaBH}_{4}$ (99\%). After $10 \mathrm{~min}$ of magnetic stirring, $4 \mathrm{mmol}$ of $\mathrm{Na}_{2} \mathrm{WO}_{4} \cdot 2 \mathrm{H}_{2} \mathrm{O}$ was added to the above solution under stirring, and the mixture thus obtained was further stirred for $1 \mathrm{~h}$. The mixed solution was then transferred to a $100-\mathrm{mL}$ Teflonlined autoclave, which was placed in a muffle furnace for hydrothermal processing at $240^{\circ} \mathrm{C}$ for $48 \mathrm{~h}$. The precipitate thus produced was collected and washed five times with deionized water and ethanol alternately, and the obtained powder was dried at $60^{\circ} \mathrm{C}$ for $10 \mathrm{~h}$. Finally, porous $2 \mathrm{D} \mathrm{WO}_{3}$ nanosheets were obtained by in-situ oxidizing the collected $\mathrm{WSe}_{2}$ nanosheets in a tube furnace under an oxygen atmosphere at $450^{\circ} \mathrm{C}$ for $3 \mathrm{~h}$.

\section{Preparation of ultrathin porous $\mathrm{MoO}_{3}$ nanosheets}

Se powder $(0.3158 \mathrm{~g})$ and $0.3 \mathrm{~g}$ of $\mathrm{NaBH}_{4}$ were poured into a beaker containing $30 \mathrm{~mL}$ of deionized water. Upon dissolution of the Se power after $10 \mathrm{~min}$ of magnetic stirring, $0.4839 \mathrm{~g}$ of $\mathrm{Na}_{2} \mathrm{MoO}_{4} \cdot 2 \mathrm{H}_{2} \mathrm{O}$ and $30 \mathrm{~mL}$ of ethanol (AR) were quickly added to the above-mentioned solution under magnetic stirring. Subsequently, the prepared precursor solution was transferred to a $100-\mathrm{mL}$ high-pressure reaction kettle, which was kept in a muffle furnace at $200^{\circ} \mathrm{C}$ for $24 \mathrm{~h}$. After the mixture was naturally cooled to room temperature, the black $\mathrm{MoSe}_{2}$ powder was collected by washing followed by centrifugation; it was then calcined in a tube furnace at $400^{\circ} \mathrm{C}$ for $3 \mathrm{~h}$ under an oxygen atmosphere. Finally, porous $2 \mathrm{D} \mathrm{MoO}_{3}$ nanosheets were obtained.

Preparation of ultrathin porous $\mathrm{Co}_{3} \mathrm{O}_{4}$ nanosheets Ultrathin porous cobalt oxide nanosheets were also obtained by calcining cobalt selenide nanosheets that was prepared by implementing the hydrothermal method. In detail, $0.0866 \mathrm{~g}$ of $\mathrm{Na}_{2} \mathrm{SeO}_{3}$ and $0.1455 \mathrm{~g}$ of $\mathrm{Co}\left(\mathrm{NO}_{3}\right)_{2}$. $6 \mathrm{H}_{2} \mathrm{O}$ were transferred to a three-necked flask containing $18 \mathrm{~mL}$ of deionized water. After stirring, the mixture thus obtained for $20 \mathrm{~min}$, and $2 \mathrm{~mL}$ of hydrazine hydrate were added to a three-necked flask under nitrogen atmosphere. The obtained mixture was then transferred to an autoclave with Teflon lining for hydrothermal processing conducted at $160^{\circ} \mathrm{C}$ for $24 \mathrm{~h}$. Once the reactor was allowed to cool to room temperature, the black ultrathin cobalt selenide nanosheets could be obtained by repeated centrifugation and ethanol washing. Finally, the porous $2 \mathrm{D} \mathrm{Co}_{3} \mathrm{O}_{4}$ nanosheets were collected after calcining the dried cobalt selenide nanosheets in an oxygen atmosphere at $600^{\circ} \mathrm{C}$ for $4 \mathrm{~h}$.

\section{Characterization}

X-ray diffraction (XRD, Philips X' Pert diffractometer) with a $\mathrm{Cu} \mathrm{Ka}$ light source was used to determine the phase of the sample. Field emission scanning electron microscopy (FESEM, FEI Sirion200) and TEM (FEI Tecnai G2 F20) were employed to investigate the morphology of the sample. Energy-dispersive X-ray spectroscopy (EDS, ULTIM MAX 100) was used to determine the elemental content of $\mathrm{WO}_{3}$ nanosheets. AFM (SPM9700, Shimadzu, Japan) was employed to determine the thickness of the nanosheets, and X-ray photoelectron spectroscopy (XPS, Kratos XSAM800 spectrometer) was used to analyze the molecular structure and atomic valence of the sample. Thermogravimetric differential scanning calorimetry (TG-DSC) was used to determine the minimum oxidation temperature for the prepared TMSe nanosheets.

\section{Gas sensor measurements and photodetector measurements}

The micro-dropping method was implemented to evenly distribute the $\mathrm{WSe}_{2}$ nanosheets dispersed in ethanol with ultrasound onto the electrode substrate. The prepared electrode substrate was dried at $80^{\circ} \mathrm{C}$ for $10 \mathrm{~min}$ to remove the ethanol and placed in a tube furnace, which was calcined under an oxygen atmosphere at $450^{\circ} \mathrm{C}$ for $3 \mathrm{~h}$, thereby preparing a $\mathrm{WO}_{3}$ device that could be used for hydrogen sulfide $\left(\mathrm{H}_{2} \mathrm{~S}\right)$ gas sensing. Gas sensing relied on a high-throughput test platform built independently by our laboratory. The prepared device was inserted into the testing chamber, and a heating voltage was applied. We controlled the concentration of the $\mathrm{H}_{2} \mathrm{~S}$ gas by adjusting the ratio of $\mathrm{H}_{2} \mathrm{~S}$ gas to dry air. Next, according to the response value formula, $S=R_{\mathrm{a}} / R_{\mathrm{g}}-1$, where $S$ is the 
response value, $R_{\mathrm{a}}$ is the resistance value of the device in the air background, and $R_{\mathrm{g}}$ is the response resistance value of the device to $\mathrm{H}_{2} \mathrm{~S}$ gas. Finally, the response of gas sensing was calculated by analyzing the resistance signal recorded by a computer. The above-mentioned device can also be used for photodetector testing. By applying to or removing from the device containing porous $2 \mathrm{D} \mathrm{WO}_{3}$ nanosheets, the uniform optical radiation $(400 \mathrm{~nm}$ peak, $5.20 \mathrm{~mW} \mathrm{~cm}^{-2}$ ), and photoelectric response data were collected with the high-throughput test platform independently developed by our laboratory.

\section{RESULTS AND DISCUSSION}

The two-step method for preparing ultrathin porous 2D TMO nanosheets is roughly depicted in Fig. 1. During the oxidation of ultrathin TMSe nanosheets prepared via the hydrothermal method in an oxygen atmosphere at a specific temperature, Se atoms in TMSe nanosheets will undergo complete oxidation to generate the corresponding stable oxide $\mathrm{SeO}_{2}$. This compound is in the gas phase at high temperature, and it will stay in the tube furnace with the oxygen flow. Taking the oxidation process of $\mathrm{WSe}_{2}$ as an example, the said reaction course is represented by the following equation [25]:

$2 \mathrm{WSe}_{2}+7 \mathrm{O}_{2} \rightarrow 2 \mathrm{WO}_{3}+4 \mathrm{SeO}_{2}$.

In order to confirm that $\mathrm{WSe}_{2}$ had been oxidized completely, we performed EDS experiments on the asprepared porous $2 \mathrm{D} \mathrm{WO}_{3}$ nanosheets. As can be evinced from Fig. S1d, the content of Se atoms in the product $\mathrm{WO}_{3}$ is 0 , and the atomic ratio of $\mathrm{O}$ to $\mathrm{W}$ is $76.69 / 23.31$, which is close to the theoretical stoichiometric ratio of
$3 / 1$.

As the in-situ oxidation of Se centers proceeds, the TMO lattice continues to grow. Since the $\mathrm{WSe}_{2}$ precursor is sufficiently thin (about $5 \mathrm{~nm}$ ), the lattice growth process of $\mathrm{WO}_{3}$ is limited to the $2 \mathrm{D}$ space, thereby thwarting the tendency of TMO nanosheets to form granular structures, and a large number of micropores form on the surface of the nanosheet as a way to release the huge surface stress associated with maintaining the original 2D morphology of the TMSe nanosheets. Through this topology transformation strategy, we successfully prepared several ultrathin porous TMO nanosheets, such as $\mathrm{WO}_{3}$, $\mathrm{MoO}_{3}$, and $\mathrm{Co}_{3} \mathrm{O}_{4}$. Details on these types of nanosheets refer to the Supplementary information.

As can be evinced from Fig. 2, SEM and TEM experiments were conducted to determine the morphologies of several TMSe nanosheet precursors. Clearly, these selenide nanosheet precursors all displayed ultrathin and dense sheet morphologies, and the edges of the said sheets were characterized by a degree of curling due to surface tension; notably, these structural features are very similar to those of reduced GO (Figs S2 and S3). TEM was also employed to observe the pore structure and lattice distribution of ultrathin porous TMO nanosheets (Figs S4 and S5). Taking ultrathin porous $\mathrm{WO}_{3}$ nanosheets as an example, the TEM image in Fig. $2 \mathrm{~d}$ indicates that the lateral size of the prepared nanosheets can reach several hundred nanometers, and this structural feature is a direct consequence of the size of the nanosheets of the precursor, $\mathrm{WSe}_{2}$. The XRD pattern reported in Fig. 2a indicates that $\mathrm{WSe}_{2}$ nanosheets are completely transformed into orthogonal phase $\mathrm{WO}_{3}$ (JCPDS No.20-1324)

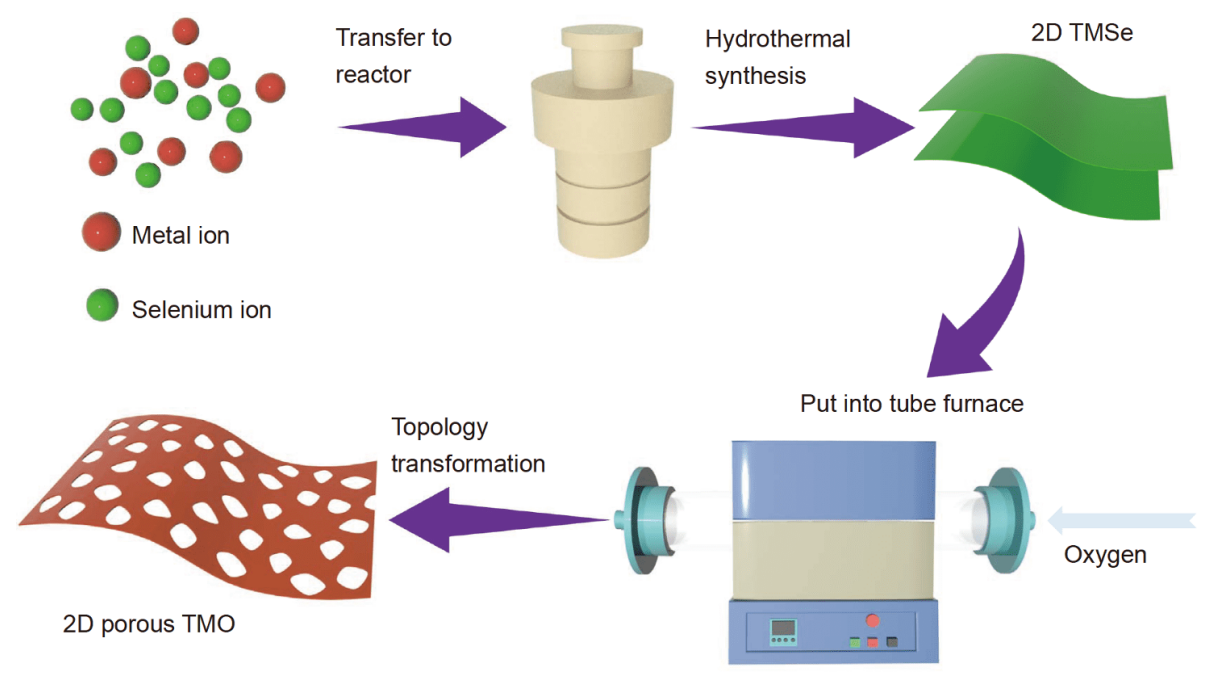

Figure 1 Schematic diagram of the two-step synthesis of the 2D porous TMO nanosheets. 

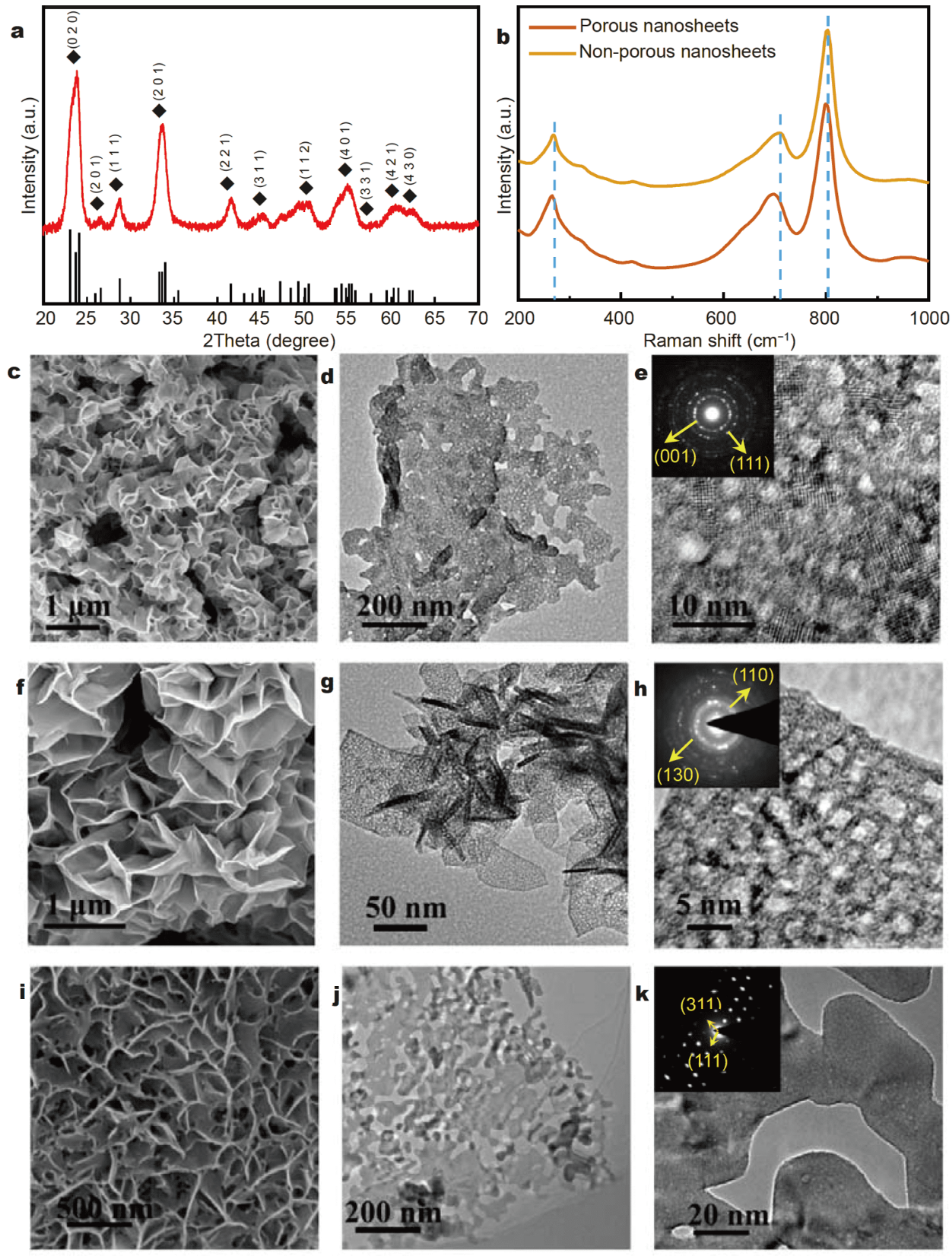

Figure 2 (a, b) XRD and Raman results of the $2 \mathrm{D}$ porous $\mathrm{WO}_{3}$ nanosheets. (c, f, i) SEM images of the ultrathin WSe $\mathrm{CoSe}_{2}$ and $\mathrm{Co}_{0.85} \mathrm{Se}$ precursors. (d, g, j) TEM images of the 2D porous $\mathrm{WO}_{3}, \mathrm{MoO}_{3}$ and $\mathrm{Co}_{3} \mathrm{O}_{4} \cdot(\mathrm{e}, \mathrm{h}, \mathrm{k})$ High-resolution TEM images and selected area electron diffraction images of the $2 \mathrm{D}$ porous $\mathrm{WO}_{3}, \mathrm{MoO}_{3}$ and $\mathrm{Co}_{3} \mathrm{O}_{4}$.

with lattice constants $a=7.384, b=7.512$, and $c=3.846$, after undergoing calcination in an oxygen atmosphere. Notably, Raman spectroscopy measurements can afford the non-destructive detection of defects in $2 \mathrm{D}$ materials [26]. Therefore, we compared the Raman spectra of smooth and porous $\mathrm{WO}_{3}$ nanosheets and found that the Raman peak position of $\mathrm{WO}_{3}$ nanosheets with a porous structure exhibits an obvious shift with respect to the positions of their counterparts for smooth $\mathrm{WO}_{3}$ nanosheets (Fig. 2b). This observation may be related to phonon softening and phonon-electron coupling induced by point defects [27]. In addition, evidence suggests that dense pore structures are evenly distributed on the surface of the $\mathrm{WO}_{3}$ nanosheets; additionally, results of 
Brunner-Emmet-Teller (BET) measurements indicate the specific surface area of the nanosheet to be about $58.9 \mathrm{~m}^{2} \mathrm{~g}^{-1}$ (Fig. S6), and the average pore diameter to be 2-4 nm. As can be evinced from Fig. $2 \mathrm{c}-\mathrm{k}$, high-resolution TEM evidence supports the data on the average pore diameter obtained by BET; moreover, the selected area electron diffraction patterns indicate that $\mathrm{WO}_{3}$ and $\mathrm{MoO}_{3}$ nanosheets prepared by this chemical topology transformation strategy are polycrystalline, while $\mathrm{Co}_{3} \mathrm{O}_{4}$ nanosheets are composed of single crystals. AFM evidence indicates the thickness of the $\mathrm{WO}_{3}$ nanosheets is about $4.6 \mathrm{~nm}$ (Fig. 3a), which is equivalent to a stack of seven $\mathrm{WO}_{3}$ monolayers [28]. The 2D ultrathin structure and the abundance of pores in nanosheets will inevitably bring about a short electron migration channel, a large exposed surface, and a wealth of active sites, which could be very beneficial to the materials' development and application to the manufacture of electronic devices.

XPS was employed to determine the chemical composition and element valence states of the ultrathin porous $\mathrm{WO}_{3}$ nanosheets (Fig. 3b, c). We found the binding energies of $\mathrm{W} 5 \mathrm{P}_{3 / 2}, \mathrm{~W} 4 \mathrm{f}_{5 / 2}$, and $\mathrm{W} 4 \mathrm{f}_{7 / 2}$ to be $41.8,38.0$, and $35.9 \mathrm{eV}$, respectively, which are basically consistent with those reported for $\mathrm{W}^{6+}$ [29]. Moreover, it is worth noting that the binding energies of $\mathrm{W} 4 \mathrm{f}_{5 / 2}$ and $\mathrm{W} 4 \mathrm{f}_{7 / 2}$ have increased offsets of $0.2-0.6 \mathrm{eV}$ compared with the standard reference of $\mathrm{WO}_{3}$ (Table S1), indicating that the charge density around the $\mathrm{W}$ atom has decreased with
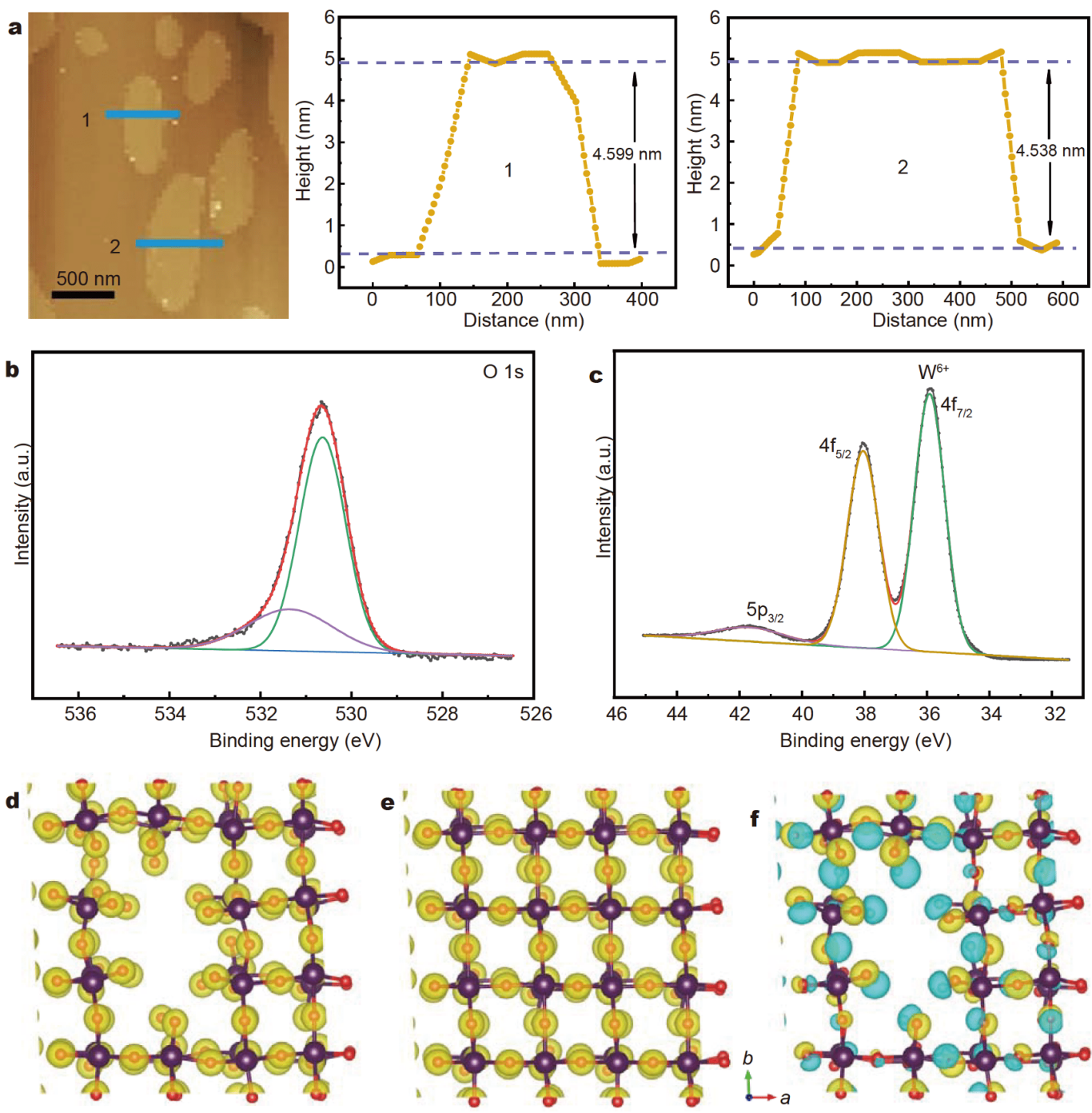

Figure 3 (a) AFM image and thickness annotation of the $2 \mathrm{D}$ porous $\mathrm{WO}_{3}$ nanosheets. (b, c) Typical XPS spectra of the $2 \mathrm{D}$ porous $\mathrm{WO}$ nanosheets: $\mathrm{O}$ peaks and $\mathrm{W}$ peaks. (d, e) Charge density distribution of the porous and non-porous $\mathrm{WO}_{3}$ nanosheets. (f) Differential charge density diagram between the porous $\mathrm{WO}_{3}$ nanosheets and the non-porous $\mathrm{WO}_{3}$ nanosheets. 
respect to the said standard; this observation may descend from an electronic rearrangement caused by the lattice distortion and chemical bond breakage. The intuitive explanation of this process is that, with the breakage of the $\mathrm{W}-\mathrm{Se}$ bond and the formation of the $\mathrm{W}-\mathrm{O}$ bond, electrons begin to transfer from the $\mathrm{W}$ atom to the $\mathrm{O}$ atom, which leads to an increase in chemical state of the $\mathrm{W}$ atom. DFT calculations were performed to estimate the value of the differential charge density of porous $\mathrm{WO}_{3}$ nanosheets and non-porous ideal $\mathrm{WO}_{3}$ nanosheets. As can be easily evinced from Fig. 3d-f, the charge transfer of $\mathrm{W}$ atoms in porous $\mathrm{WO}_{3}$ nanosheets is significantly enhanced with respect to non-porous $\mathrm{WO}_{3}$ nanosheets. Compared with the ideal non-porous $\mathrm{WO}_{3}$ nanosheet, the charge of the six tungsten atoms at the pore edge of a porous $\mathrm{WO}_{3}$ nanosheet transfers 0.14 more electron than non-porous $\mathrm{WO}_{3}$. In addition, the average charge of $\mathrm{W}$ atoms in porous $\mathrm{WO}_{3}$ nanosheets is reduced by $0.05 \mathrm{e}$ with respect to non-porous $\mathrm{WO}_{3}$ nanosheets (Fig. S7). The calculated value of the Bader charge further supports the enhancement of the charge transfer associated with the presence of pores; notably, this enhancement often means an increase in reactivity [27,30-31], which is beneficial to the potential applications of the obtained porous materials.

In order to test the application performance of such ultrathin porous TMO nanosheets, we conducted $\mathrm{H}_{2} \mathrm{~S}$ gas-sensing tests with ultrathin porous $\mathrm{WO}_{3}$ nanosheets as a typical example. $\mathrm{H}_{2} \mathrm{~S}$ is well known to be a good electron donor, and we found the resistance of the $\mathrm{WO}_{3}$ nanosheet sensor to be significantly reduced when the sensor was in contact with $\mathrm{H}_{2} \mathrm{~S}$ gas; moreover, the said resistance quickly and completely recovered its original value when the flow of $\mathrm{H}_{2} \mathrm{~S}$ gas was discontinued, which is the typical behavior expected from n-type semiconductors. We compared the responses of $\mathrm{WO}_{3}$ nanosheets to $\mathrm{H}_{2} \mathrm{~S}$ gas at a concentration of $2 \mathrm{ppm}$ at different temperatures and found that, as the temperature gradually increased to $160^{\circ} \mathrm{C}$, the response value continued to increase and reached a peak at $160^{\circ} \mathrm{C}$; above this temperature, however, the response value gradually decreased as the temperature increased. Therefore, we determined the best working temperature for $\mathrm{WO}_{3}$ nanosheets to be $160^{\circ} \mathrm{C}$. As can be evinced from Fig. 4,
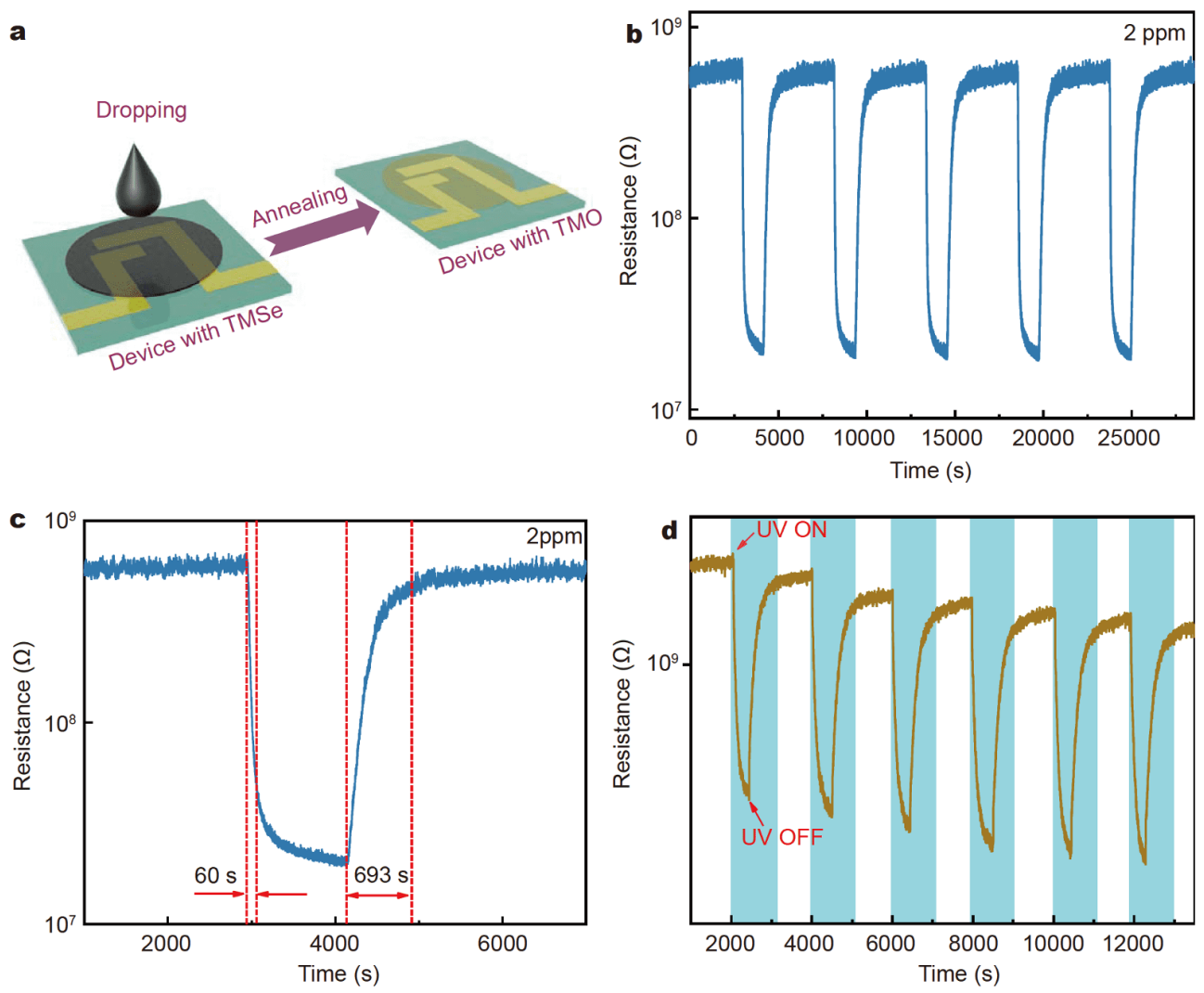

Figure 4 (a) Schematic diagram of the device prepared by dripping-annealing. (b, c) The cycle stability curve and response/recovery curve of the sensor to $2 \mathrm{ppm}$ hydrogen sulfide at $160^{\circ} \mathrm{C}$. (d) Photoelectric response/recovery curve of the $\mathrm{WO}_{3}$ device. 
five repeated measurements indicated that the average

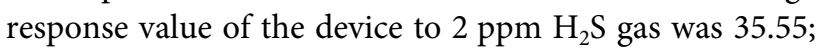
additionally, the values for the response/recovery times measured in the five tests were basically the same, with the resistance value returning to the baseline value every time. Such gas-sensing performance was observed to be superior to those reported in the literature for $\mathrm{H}_{2} \mathrm{~S}$ gas sensors [32-35]; these results undoubtedly indicate that the porous $2 \mathrm{D} \mathrm{WO}_{3}$ nanosheets, or other TMO nanosheets, have great potential as gas sensors. Similarly, we also found that the value of the light-dark resistance ratio of $\mathrm{WO}_{3}$ nanosheets was $317 \%$ when used in photodetectors, and it can not only recover to the baseline stably, but also display an extremely high response/recovery speed, suggesting that $\mathrm{WO}_{3}$ nanosheets also have the potential to be utilized as optoelectronic devices.

\section{CONCLUSION}

In summary, based on a simple topology transformation strategy, we synthesized several kinds of porous 2D TMO nanosheets, such as $\mathrm{WO}_{3}, \mathrm{MoO}_{3}$, and $\mathrm{Co}_{3} \mathrm{O}_{4}$, by oxidizing the corresponding TMSe nanosheets. In fact, the use of ultrathin TMSe nanosheets limits the lattice growth process of TMO nanosheets to the $2 \mathrm{D}$ space, while the formation of pores affords the means to release the huge stress associated with crystal lattice growth, thereby maintaining a complete 2D morphology and avoiding the fracture and granulation of nanosheets during the oxidation process. We also found that the as-synthesized porous $2 \mathrm{D} \mathrm{WO}_{3}$ nanosheets exhibit excellent $\mathrm{H}_{2} \mathrm{~S}$ gas sensitivity and photoelectric performance; notably, the performance of this material in other fields is also worth investigating in the future. Furthermore, the range of suitable precursors that can be utilized in the described topology transformation strategy may be extended to include other ultrathin TMSe nanosheets or even other ultrathin transition metal chalcogenide nanosheets.

\section{Received 23 December 2020; accepted 7 February 2021;} published online 26 April 2021

1 Novoselov KS, Geim AK, Morozov SV, et al. Electric field effect in atomically thin carbon films. Science, 2004, 306: 666-669

2 Haque F, Daeneke T, Kalantar-Zadeh K, et al. Two-dimensional transition metal oxide and chalcogenide-based photocatalysts. Nano-Micro Lett, 2018, 10: 23

$3 \mathrm{Lu} \mathrm{Q}, \mathrm{Yu} \mathrm{Y}, \mathrm{Ma} \mathrm{Q}$, et al. 2D transition-metal-dichalcogenidenanosheet-based composites for photocatalytic and electrocatalytic hydrogen evolution reactions. Adv Mater, 2016, 28: 1917-1933

4 Niu P, Zhang L, Liu G, et al. Graphene-like carbon nitride nanosheets for improved photocatalytic activities. Adv Funct Mater, 2012, 22: 4763-4770
5 Wang Q, O'Hare D. Recent advances in the synthesis and application of layered double hydroxide (LDH) nanosheets. Chem Rev, 2012, 112: 4124-4155

6 Zhang H. Ultrathin two-dimensional nanomaterials. ACS Nano, 2015, 9: 9451-9469

7 Novoselov KS, Mishchenko A, Carvalho A, et al. 2D materials and van der Waals heterostructures. Science, 2016, 353: aac9439

8 Ji H, Zeng W, Li Y. Gas sensing mechanisms of metal oxide semiconductors: a focus review. Nanoscale, 2019, 11: 22664-22684

9 Kumar R, Liu X, Zhang J, et al. Room-temperature gas sensors under photoactivation: from metal oxides to $2 \mathrm{D}$ materials. NanoMicro Lett, 2020, 12: 164

10 Dou Y, Liao T, Ma Z, et al. Graphene-like holey $\mathrm{Co}_{3} \mathrm{O}_{4}$ nanosheets as a highly efficient catalyst for oxygen evolution reaction. Nano Energy, 2016, 30: 267-275

11 Mei J, Liao T, Kou L, et al. Two-dimensional metal oxide nanomaterials for next-generation rechargeable batteries. Adv Mater, 2017, 29: 1700176

12 Kang Y, Xie H, Liu D, et al. Facile mass production of self-supported two-dimensional transition metal oxides for catalytic applications. Chem Commun, 2019, 55: 11406-11409

13 Ji F, Ren $\mathrm{X}$, Zheng $\mathrm{X}$, et al. $2 \mathrm{D}-\mathrm{MoO}_{3}$ nanosheets for superior gas sensors. Nanoscale, 2016, 8: 8696-8703

14 Zhao C, Zhang H, Si W, et al. Mass production of two-dimensional oxides by rapid heating of hydrous chlorides. Nat Commun, 2016, 7: 12543

15 Peng L, Xiong P, Ma L, et al. Holey two-dimensional transition metal oxide nanosheets for efficient energy storage. Nat Commun, 2017, 8: 15139

16 Sun Z, Liao T, Dou Y, et al. Generalized self-assembly of scalable two-dimensional transition metal oxide nanosheets. Nat Commun, 2014, 5: 3813

17 Zhao H, Zhu Y, Li F, et al. A generalized strategy for the synthesis of large-size ultrathin two-dimensional metal oxide nanosheets. Angew Chem Int Ed, 2017, 56: 8766-8770

18 Tan C, Cao X, Wu XJ, et al. Recent advances in ultrathin twodimensional nanomaterials. Chem Rev, 2017, 117: 6225-6331

$19 \mathrm{Xu} \mathrm{K}, \mathrm{Li} \mathrm{N}$, Zeng D, et al. Interface bonds determined gas-sensing of $\mathrm{SnO}_{2}-\mathrm{SnS}_{2}$ hybrids to ammonia at room temperature. ACS Appl Mater Interfaces, 2015, 7: 11359-11368

$20 \mathrm{Wu}$ Y, Cao T, Wang R, et al. A general strategy for the synthesis of two-dimensional holey nanosheets as cathodes for superior energy storage. J Mater Chem A, 2018, 6: 8374-8381

21 Zhang C, Huang Y, Yu Y, et al. Sub-1.1 nm ultrathin porous CoP nanosheets with dominant reactive $\{200\}$ facets: a high mass activity and efficient electrocatalyst for the hydrogen evolution reaction. Chem Sci, 2017, 8: 2769-2775

22 Liu CC, Song JM, Zhao JF, et al. Facile synthesis of tremelliform $\mathrm{Co}_{0.85}$ Se nanosheets: An efficient catalyst for the decomposition of hydrazine hydrate. Appl Catal B-Environ, 2012, 119-120: 139-145

23 Wang $\mathrm{X}$, Chen $\mathrm{Y}$, Qi F, et al. Interwoven $\mathrm{WSe}_{2} / \mathrm{CNTs}$ hybrid network: A highly efficient and stable electrocatalyst for hydrogen evolution. Electrochem Commun, 2016, 72: 74-78

24 Wang Y, Zhang F, Wang Q, et al. Hierarchical $\mathrm{MoSe}_{2}$ nanoflowers as novel nanocarriers for NIR-light-mediated synergistic photothermal/dynamic and chemo-therapy. Nanoscale, 2018, 10: 1453414545

25 Liu W, Gu D, Li X. Ultrasensitive $\mathrm{NO}_{2}$ detection utilizing mesoporous $\mathrm{ZnSe} / \mathrm{ZnO}$ heterojunction-based chemiresistive-type sensors. ACS Appl Mater Interfaces, 2019, 11: 29029-29040 
Pimenta MA, Dresselhaus G, Dresselhaus MS, et al. Studying disorder in graphite-based systems by Raman spectroscopy. Phys Chem Chem Phys, 2007, 9: 1276-1290

27 Liu Y, Cheng $\mathrm{M}, \mathrm{He} \mathrm{Z}$, et al. Pothole-rich ultrathin $\mathrm{WO}_{3}$ nanosheets that trigger $\mathrm{N} \equiv \mathrm{N}$ bond activation of nitrogen for direct nitrate photosynthesis. Angew Chem Int Ed, 2019, 58: 731-735

28 Liang L, Zhang J, Zhou Y, et al. High-performance flexible electrochromic device based on facile semiconductor-to-metal transition realized by $\mathrm{WO}_{3} \cdot 2 \mathrm{H}_{2} \mathrm{O}$ ultrathin nanosheets. Sci Rep, 2013, 3: 1936

29 Hu X, Wang X, Zhou T, et al. Insight into highly selective dimethyl trisulfide detection based on $\mathrm{WO}_{3}$ nanorod bundles with exposed (002) facets. Sens Actuat B-Chem, 2020, 305: 127538

30 Wang W, Zhu YB, Wen Q, et al. Modulation of molecular spatial distribution and chemisorption with perforated nanosheets for ethanol electro-oxidation. Adv Mater, 2019, 31: 1900528

31 Kim Y, Kwon KC, Kang S, et al. Two-dimensional $\mathrm{NbS}_{2}$ gas sensors for selective and reversible $\mathrm{NO}_{2}$ detection at room temperature. ACS Sens, 2019, 4: 2395-2402

32 Wang C, Zhang Y, Sun X, et al. Preparation of Pd/PdO loaded $\mathrm{WO}_{3}$ microspheres for $\mathrm{H}_{2} \mathrm{~S}$ detection. Sens Actuat B-Chem, 2020, 321: 128629

33 Ramgir NS, Goyal CP, Sharma PK, et al. Selective $\mathrm{H}_{2} \mathrm{~S}$ sensing characteristics of $\mathrm{CuO}$ modified $\mathrm{WO}_{3}$ thin films. Sens Actuat BChem, 2013, 188: 525-532

34 Boepple $\mathrm{M}$, Zhu Z, Hu X, et al. Impact of heterostructures on hydrogen sulfide sensing: Example of core-shell $\mathrm{CuO} / \mathrm{CuFe}{ }_{2} \mathrm{O}_{4}$ nanostructures. Sens Actuat B-Chem, 2020, 321: 128523

35 Wang C, Jiang L, Wang J, et al. Mixed potential type $\mathrm{H}_{2} \mathrm{~S}$ sensor based on stabilized zirconia and $\mathrm{C}_{2} \mathrm{SnO}_{4}$ sensing electrode for halitosis monitoring. Sens Actuat B-Chem, 2020, 321: 128587

Acknowledgements This work was supported by the National Natural Science Foundation of China (61971204 and 51902114). The authors are very grateful to the technical support from the Analytic Testing Center for SEM, TEM, XPS, Raman spectroscopy measurements, and the State Key Laboratory of Materials and Processing Die \& Mould Technology of HUST for the XRD measurements.

Author contributions Li X performed the experiments and wrote the paper with the support from Zeng D. Qin Z and Hu X contributed to the data processing and theoretical analysis. Zhang $\mathrm{J}$ and $\mathrm{Wu} \mathrm{J}$ contributed to the characterization of the materials. The calculation based on the DFT was completed with the assistance of Yang H. Xie C contributed to the photodetection.

Conflict of interest The authors declare no conflict of interest.

Supplementary information Supporting data are available in the online version of the paper.

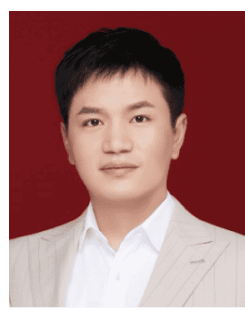

Xiang Li is currently a $\mathrm{PhD}$ candidate at the School of Materials Science and Engineering, Huazhong University of Science and Technology. His research interests include $2 \mathrm{D}$ materials and their applications in gas sensing.

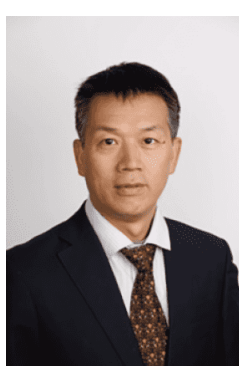

Dawen Zeng received his $\mathrm{PhD}$ from Huazhong University of Science and Technology in 1998. $\mathrm{He}$ is currently a professor at the School of Materials Science and Engineering, Huazhong University of Science and Technology. He has devoted himself to the research of functional materials for many years, and his research interests include 2D materials, metal oxide semiconductors, flexible electronic devices, and gas sensors.

\section{一种基于化学拓扑变换合成多孔二维过渡金属氧 化物纳米片的通用策略}

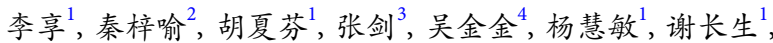
曾大文 ${ }^{{ }^{*}}$

摘要 二维(2D)过渡金属氧化物(TMO) 由于其丰富的活性位点, 快 速的离子传输速率和较短的载流子迁移距离引起了广泛关注. 但 是，当前的合成策略通常局限于本质分层的化合物或基于牺牲模 板的方法. 本文中, 我们报道了一种通过相应过渡金属硒化物的化 学拓扑转移制备超薄二维多孔TMO纳米片的通用策略. 值得注意 的是，所制备的2D TMO纳米片不仅可以完美地继承过渡金属硒化 物的 $2 \mathrm{D}$ 拓扑结构, 而且由于晶格生长中的应力释放产生了大量的 孔洞结构. 此外, 我们基于原位氧化相应的超薄 $\mathrm{WSe}_{2}, \mathrm{MoSe}_{2}$ 和 $\mathrm{Co}_{0.85} \mathrm{Se}$ 纳米片, 成功制备了多孔超薄 $\mathrm{WO}_{3}, \mathrm{MoO}_{3}$ 和 $\mathrm{Co}_{3} \mathrm{O}_{4}$ 纳米片. 所制备的多孔二维 $\mathrm{WO}_{3}$ 纳米片出色的气敏性能和光探测性能进一 步表明了该制备策略具有广阔的应用前景. 